\title{
Commentary: Forgotten no more
}

Sameer A. Hirji, MD, MPH, and

Tsuyoshi Kaneko, MD

The tricuspid regurgitation (TR) management landscape continues to evolve substantially. We read with great interest the Expert Opinion piece by Chikwe and Megna ${ }^{1}$ in this issue of the Journal. We congratulate the authors for providing a comprehensive-yet-concise synthesis of various aspects related to concomitant tricuspid valve repair (TVr), including anatomy, pathophysiology, and detailed rationale for surgery. This review is relevant, as there remains a substantial controversy in the approach to concomitant TR and variations in clinical practice. The authors propose that an aggressive approach to concomitant TR may be justified by the current consensus guidelines, emerging evidence from the Society of Thoracic Surgeon's database, which demonstrates improved tricuspid valve surgery outcomes over time, ${ }^{2}$ and the minimal incremental risk of concomitant $\mathrm{TVr}$ on overall mortality or morbidity. ${ }^{3}$

There are several points that we would like to highlight. First, TR remains under-recognized despite a high epidemiologic prevalence of TR. ${ }^{4,5}$ Although concomitant TVr appears beneficial, ${ }^{3}$ it is often not performed at the time of surgery, likely because of the notion that TR may recover upon successful elimination of the mitral regurgitation. The authors methodically dismiss this perception, especially for patients with severe TR and dilated annulus. In addition, the effect of residual TR also has detrimental long-term consequences, and we must be

From the Division of Cardiac Surgery, Department of Surgery, Brigham and Women's Hospital, Harvard Medical School, Boston, Mass.

Disclosures: Dr Kaneko reported consultant/speaker: Edwards Lifesciences, Medtronic, Abbott Structural Heart, Baylis Medical, and 4C Medical. Dr Hirji reported no conflicts of interest.

The Journal policy requires editors and reviewers to disclose conflicts of interest and to decline handling or reviewing manuscripts for which they may have a conflict of interest. The editors and reviewers of this article have no conflicts of interest.

Received for publication May 28, 2020; revisions received May 28, 2020; accepted for publication June 2, 2020; available ahead of print July 8, 2020.

Address for reprints: Tsuyoshi Kaneko, MD, Division of Cardiac Surgery, Brigham and Women's Hospital, 15 Francis St, Boston, MA, 02115 (E-mail: tkaneko2@ bwh.harvard.edu).

JTCVS Open 2020;3:62-3

2666-2736

Copyright (C) 2020 The Authors. Published by Elsevier Inc. on behalf of The American Association for Thoracic Surgery. This is an open access article under the CC BY-NCND license (http://creativecommons.org/licenses/by-nc-nd/4.0/).

https://doi.org/10.1016/j.xjon.2020.06.002

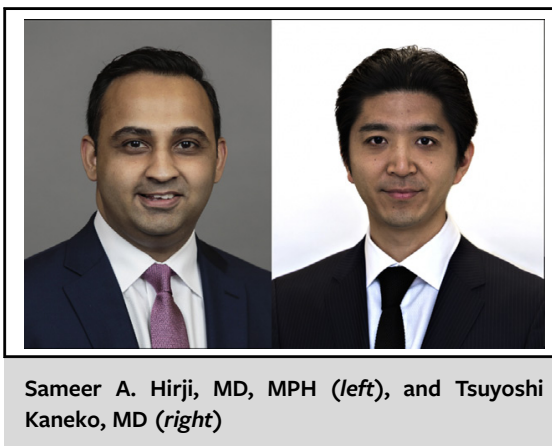

CENTRAL MESSAGE

The under-recognition and/or undertreatment of concomitant tricuspid regurgitation at the time of original cardiac surgery can lead to detrimental longterm consequences if not addressed.

cognizant not to leave behind clinically significant TR at the time of the original mitral valve surgery.

The second point is the delayed presentation of TR leading to end-organ failure. Undertreatment at the time of original cardiac surgery or delayed referral to the surgeons has led to these patients presenting with TR grades that are beyond severe, which in some cases is in the spectrum of "massive" to "torrential" TR based on a recently proposed grading scheme. ${ }^{6}$ These patients with "massive" to "torrential" TR have irreversible renal or hepatic failure and will not be amenable to surgical treatment. We must treat these patients before they reach this state, ideally at the time of the original operation.

Several unanswered questions remain. What is the best echocardiographic measurement that predicts outcomes in patients with TR? We need better measures beyond the annular diameter of $40 \mathrm{~mm}$. What is the role of tricuspid valve replacement when the residual TR is expected? What is the future of transcatheter therapies in this field? One undeniable fact is that the tricuspid valve is no longer the "forgotten valve." The Cardiothoracic Surgical Trials Network just completed enrollment for their randomized clinical trial, which aims to evaluate the efficacy of concurrent $\mathrm{TVr}$ during mitral surgery with results expected in $2023 .^{7}$ There has been a spotlight on isolated tricuspid valve surgery. ${ }^{8}$ Importantly, our focus on the tricuspid valve management should continue, and cardiac surgeons should 
not "forget" to treat concomitant TR at the time of mitral valve surgery.

\section{References}

1. Chikwe J, Megna D. Rationale and surgical strategy for concomitant tricuspid repair. J Thorac Cardiovasc Surg Open. 2020;3:52-61.

2. Kilic A, Saha-Chaudhuri P, Rankin JS, Conte JV. Trends and outcomes of tricuspid valve surgery in North America: an analysis of more than 50,000 patients from the Society of Thoracic Surgeons database. Ann Thorac Surg. 2013;96:1546-52; discussion 1552.

3. Badhwar V, Rankin JS, He M, Jacobs JP, Furnary AP, Fazzalari FL, et al. Performing concomitant tricuspid valve repair at the time of mitral valve operations is not associated with increased operative mortality. Ann Thorac Surg. 2017;103:587-93.
4. Nath J, Foster E, Heidenreich PA. Impact of tricuspid regurgitation on long-term survival. J Am Coll Cardiol. 2004;43:405-9.

5. Neuhold S, Huelsmann M, Pernicka E, Graf A, Bonderman D, Adlbrecht C, et al Impact of tricuspid regurgitation on survival in patients with chronic heart failure: unexpected findings of a long-term observational study. Eur Heart J. 2013;34: 844-52.

6. Hahn RT, Thomas JD, Khalique OK, Cavalcante JL, Praz F, Zoghbi WA. Imaging assessment of tricuspid regurgitation severity. JACC Cardiovasc Imaging. 2019; 12:469-90.

7. Gelijns AL. Evaluating the benefit of concurrent tricuspid valve repair during mitral surgery. Clinical Trial - NCT02675244. Available at: https://www. clinicaltrials.gov/ct2/show/NCT02675244. Accessed May 20, 2020.

8. Axtell AL, Bhambhani V, Moonsamy P, Healy EW, Picard MH, Sundt TM III et al. Surgery does not improve survival in patients with isolated severe tricuspid regurgitation. J Am Coll Cardiol. 2019;74:715-25. 